\title{
All-passive phase locking of a compact Er:fiber laser system
}

\author{
Günther Krauss, ${ }^{1}$ David Fehrenbacher, ${ }^{1}$ Daniele Brida, ${ }^{1,2}$ Claudius Riek, ${ }^{1}$ Alexander Sell, ${ }^{1}$ \\ Rupert Huber, ${ }^{1}$ and Alfred Leitenstorfer ${ }^{1, *}$ \\ ${ }^{1}$ Department of Physics and Center for Applied Photonics, University of Konstanz, D-78457 Konstanz, Germany \\ ${ }^{2}$ Institute for Photonics and Nanotechnologies-Consiglio Nazionale delle Ricerche (IFN-CNR), Dipartimento di Fisica, \\ Politecnico di Milano, Piazza Leonardo da Vinci 32, 20133 Milano, Italy \\ *Corresponding author: alfred.leitenstorfer@uni-konstanz.de
}

Received November 30, 2010; revised January 10, 2011; accepted January 11, 2011; posted January 14, 2011 (Doc. ID 138989); published February 10, 2011

\begin{abstract}
A passively phase-locked laser source based on compact femtosecond Er:fiber technology is introduced. The carrierenvelope offset frequency is set to zero via difference frequency generation between a soliton at a wavelength of $2 \mu \mathrm{m}$ and a dispersive wave at $860 \mathrm{~nm}$ generated in the same highly nonlinear fiber. This process results in a broadband output centered at $1.55 \mu \mathrm{m}$. Subsequently, the $40 \mathrm{MHz}$ pulse train seeds a second Er:fiber amplifier, which boosts the pulse energy up to $8 \mathrm{~nJ}$ at a duration of $125 \mathrm{fs}$. Excellent phase stability is demonstrated via f-to-2f spectral interferometry. (c) 2011 Optical Society of America

OCIS codes: $320.7160,140.3510,190.7110$.
\end{abstract}

Generation of laser pulses containing few optical cycles with a shot-to-shot reproducibility of the electric field profile represents a central challenge in ultrafast optics. In fact, pulses featuring a stable carrier-envelope phase (CEP) are crucial in light-matter interaction processes directly driven by the electric field rather than the pulse intensity. Therefore, phase locking is fundamental in highfield applications such as above-threshold ionization [1], ultrafast streaking [2], and high harmonic generation for attosecond pulse synthesis [3]. Further applications are found in precision metrology with femtosecond frequency combs [4]], where the cancellation of the CEP slip is equivalent to setting the carrier-envelope offset frequency $f_{\text {CEO }}$ to zero.

Absolute control of the CEP has been widely demonstrated with systems based on Ti:sapphire, where reliable active and passive stabilization schemes can be implemented. For amplified systems in the kilohertz regime, the oscillator $f_{\text {CEO }}$ is typically locked to a harmonic of the amplifier repetition rate. Active CEP stabilization, however, requires cumbersome feedback loops. Locking of $f_{\text {CEO }}=0$ has been demonstrated utilizing an acoustooptic modulator either in a modified f-to-2f scheme [5] or by frequency shifting of the comb outside the cavity [6].

Passive control relies on difference frequency generation (DFG) between two pulses sharing the same carrierenvelope phase $\varphi$. In this case, an output results with a constant phase $\varphi_{\mathrm{DFG}}=\varphi-\varphi-\pi / 2=$ const [7]. This technique is typically exploited at kilohertz repetition rates where the phase-stable seed can be boosted efficiently with optical parametric amplification (OPA). The phaselocked seed may be provided via (i) the idler wave generated in an OPA process [7], (ii) DFG between two OPAs at different wavelengths [8], or (iii) DFG between the spectral wings of a single pulse broadened by supercontinuum generation [9]. The implementation of all-passive CEP control at oscillator repetition rates has previously been hampered by small seed pulse energies which are difficult to amplify with Ti:sapphire technology [10]. Effective operation has been achieved only by optical parametric amplification schemes [11, 12$]$.
In this Letter, we present a laser system that enables passive CEP locking of femtosecond Er:fiber laser technology. Our scheme allows for the generation of pulses with a controlled profile of the electric field at the full repetition rates of oscillators and with easily scalable pulse energy. The compact setup is sketched in Fig. 1. The Er:oscillator relies exclusively on polarizationmaintaining fibers. It is mode locked at a repetition rate of $40 \mathrm{MHz}$ with a saturable absorber mirror based on InGaAs quantum wells. The pulse train seeds a single-pass Er:fiber amplifier [13] that is pumped from both sides with $750 \mathrm{~mW}$ of average power at a wavelength of $974 \mathrm{~nm}$. The $8 \mathrm{~nJ}$ pulses are then compressed to a duration of 125 fs by a pair of Si prisms and coupled into an assembly of a standard telecom fiber of a length of $14.5 \mathrm{~cm}$ that is directly spliced to a 13-mm-long highly nonlinear germanosilicate bulk fiber (HNF) [14]. The spectrum generated in this stage is shown in Fig. 2(a). Setting the correct prechirp with the Si compressor [15], we tune the dispersive wave to a center wavelength of $860 \mathrm{~nm}$, while the corresponding soliton resides at $1950 \mathrm{~nm}$. Note that the two components share the same CEP because they are generated via third-order processes in the HNF [16].

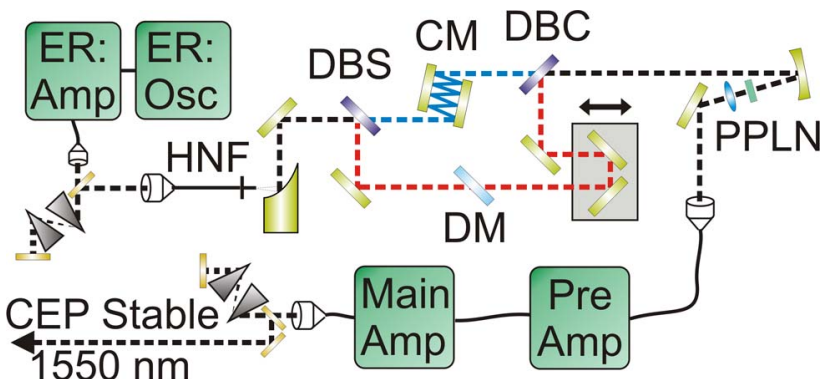

Fig. 1. (Color online) Schematic setup of the laser system. Er:Osc, femtosecond Er:fiber oscillator; Er:Amp, erbium-doped fiber amplifier; HNF, highly nonlinear germanosilicate fiber for frequency conversion; DBS, dichroic beam splitter; DBC, dichroic beam combiner; CM, chirped mirrors for pulse compression; DM, dielectric mirror for $1550 \mathrm{~nm}$; PPLN, periodically poled lithium niobate crystal for difference frequency generation; Pre Amp, preamplifier; Main Amp, main amplifier. 
Subsequently, the dispersive wave and the soliton are separated via a dichroic beam splitter reflecting at wavelengths above $1550 \mathrm{~nm}$. The branch at $860 \mathrm{~nm}$ is compressed to a pulse duration of $30 \mathrm{fs}$ by eight reflections on two chirped mirrors with a group velocity dispersion of $-130 \mathrm{fs}^{2}$ per bounce. This stage also removes residual radiation around the fundamental wavelength of $1550 \mathrm{~nm}$. The pulse duration of the soliton amounts to $40 \mathrm{fs}$. It is sent over a variable delay line and superimposed with the dispersive wave with a dichroic beam combiner. The fundamental component in this branch is filtered out by transmission through two thin dielectric mirrors reflecting from 1450 to $1600 \mathrm{~nm}$. After recombination, the average powers of the dispersive wave and the soliton are $16 \mathrm{~mW}$ and $25 \mathrm{~mW}$, respectively. We focus both components into a 2-mm-long periodically poled lithium niobate crystal via a spherical mirror with a focal length of $25 \mathrm{~mm}$. The nonlinear crystal exhibits a fan-out design. A poling period ranging from $21 \mu \mathrm{m}$ to $35 \mu \mathrm{m}$ allows for fine tuning of the output wavelength. As explained previously, the pulses generated via DFG automatically emerge with passive CEP stability due to the phase cancellation within the same frequency comb [7,17-19]. The average power obtained at a center wavelength of $1550 \mathrm{~nm}$ amounts to $1.9 \mathrm{~mW}$. A spectrum of this pulse train is shown as a gray solid curve in Fig. 2(b).

We reamplify these pulses in order to obtain output parameters that allow for efficient driving of nonlinearities in both fiber-optic devices and free-space components. To this end, we couple an average power of $840 \mu \mathrm{W}$ into a polarization-maintaining single-mode fiber that guides the DFG pulses into a preamplifier stage. This section increases the average power to $12 \mathrm{~mW}$. Subsequently, the output is split into six parallel ports via fiber-
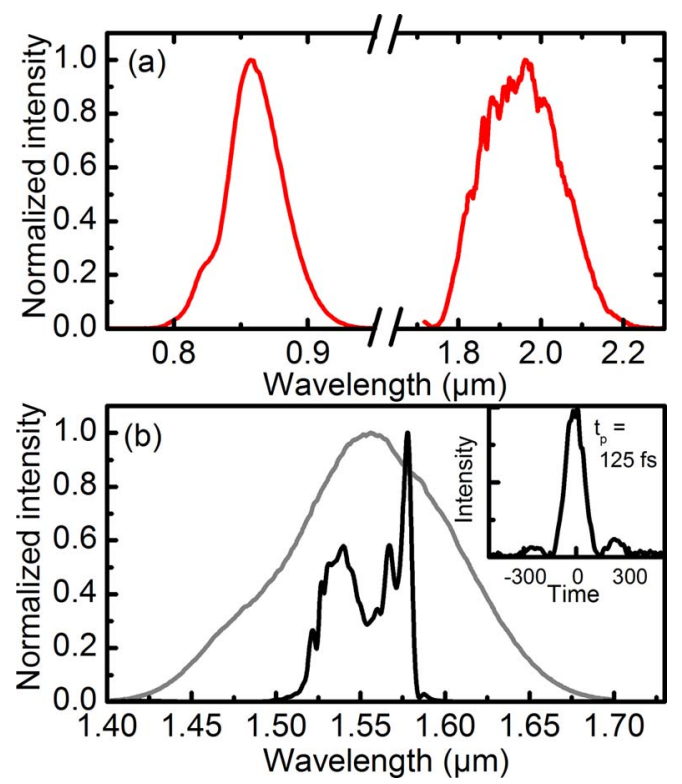

Fig. 2. (Color online) (a) Spectra of the dispersive wave (left) and soliton (right) generated by the highly nonlinear fiber assembly. Note the break in the horizontal axis. (b) Spectra of the difference frequency generated by the two spectral components depicted in (a) (gray curve) and the output of the main amplifier (black curve). The inset shows the temporal profile of the compressed pulse retrieved by a FROG measurement. based couplers. This stage enables implementation of multiple parallel amplifiers for future extensions of the experiment. In the present work, we use one branch to seed a femtosecond Er:amplifier analogous to the device located directly after the oscillator. The output spectrum obtained following this step is shown as a black solid line in Fig. 2(b). A frequency-resolved optical gating (FROG) measurement yields a duration of $125 \mathrm{fs}$ of the compressed 8nJ pulse [see inset in Fig. 2(b)].

We now analyze the CEP stability by means of an f-to-2f spectral interferometer [20]. A second highly nonlinear fiber assembly is pumped with the amplified DFG pulses in order to generate a soliton centered at $1800 \mathrm{~nm}$ and a dispersive wave at $900 \mathrm{~nm}$. After this $\mathrm{HNF}$ we collimate the ultrabroadband spectrum and focus into a 2-mm-thick $\beta$ barium borate (BBO) crystal cut at $20^{\circ}$ for type I phase matching. Here, the second harmonic (SHG) of the soliton is generated with a polarization perpendicular to the dispersive wave. We subsequently adjust the ratio of the intensities of the SHG and the dispersive wave with a polarizer. The optimum superposition is then coupled into a spectrometer equipped with a CCD array. Figure $\underline{3}$ shows the resulting interference patterns. A $4 \mathrm{~ms}$ integration time of the CCD implies that the acquired fringes are averaged over 160,000 laser pulses. Therefore, the modulation depth of $66 \%$ indicates a high degree of CEP stability on short time scales. To make sure that the fringes prove the CEP stability, we inserted different slabs of SF66 glass before generation of the second harmonic in the BBO crystal. Because of different group velocities of the soliton and dispersive wave, this material path corresponds to a delay between the two pulses and results in a different periodicity of the interference fringes. The two patterns are acquired with $2 \mathrm{~mm}$ [Fig. 3(a)] and $5 \mathrm{~mm}$ of SF66 [Fig. 3(b)], respectively. As a consequence, one would

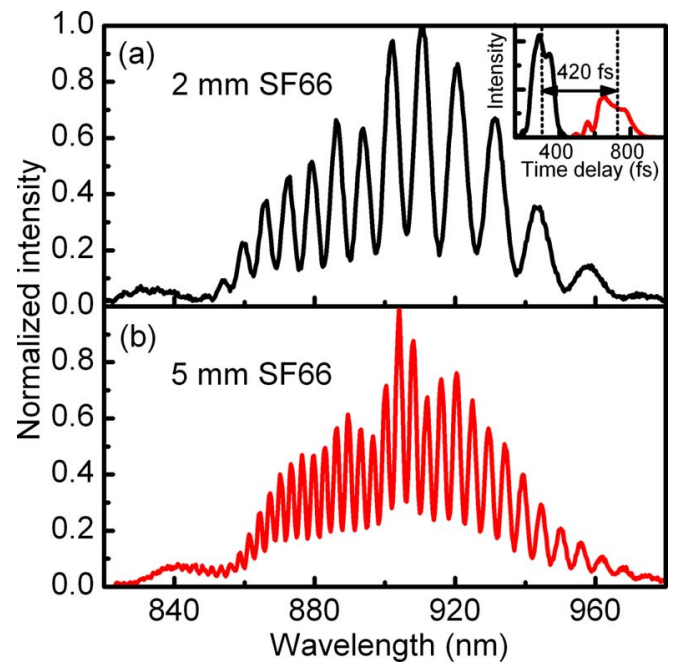

Fig. 3. (Color online) Spectral interference between the dispersive wave and the SHG of the soliton from the second HNF. (a) and (b) Fringe patterns corresponding to different delays between the fundamental and the SHG caused by different passage through SF66 glass (2 and $5 \mathrm{~mm}$, respectively) before the SHG. The inset shows Fourier transforms of each spectrum. The dotted lines denote a time interval of $420 \mathrm{fs}$, corresponding to the expected group delay caused by the $3 \mathrm{~mm}$ difference of inserted SF66. 


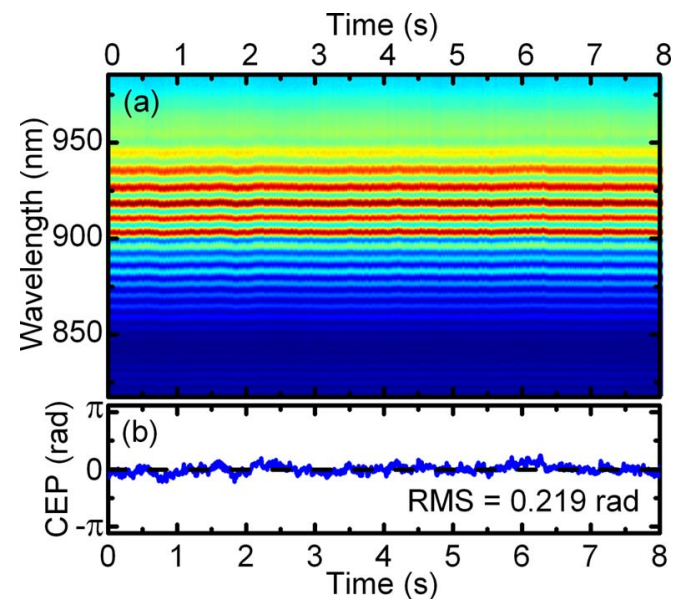

Fig. 4. (Color online) Long-term stability of the passive phase lock. A sequence of 1000 interference spectra collected over a time interval of $8 \mathrm{~s}$ is shown in a color-coded map in (a). Each spectrum was measured with an integration time of $4 \mathrm{~ms}$. The phase slip in time deduced from each individual spectrum is depicted versus time in (b). The measured RMS deviation amounts to an angle of $0.219 \mathrm{rad}$.

expect a delay of $420 \mathrm{fs}$ between the pulses and a corresponding difference of the fringe period. This estimate is in perfect agreement with our measurement, as shown in the inset of Fig. $\underline{3}$, via Fourier transform of both interference spectra.

To characterize the long-term stability of the system, we take a series of 1000 spectra measured over an interval of $8 \mathrm{~s}$ [Fig. 4(a)]. The stability of the fringe pattern is excellent and only minor deviations are discernible as a function of time. By performing a Fourier transform of each spectrum and comparing the CEP values, we calculate the root-mean-square (RMS) deviation to be as low as $0.219 \mathrm{rad}$ [see Fig. 4(b)]. With minor efforts toward isolation of the system from environmental fluctuations such as a completely closed encasement, even this result may be improved easily.

In conclusion, we have demonstrated all-passive CEP stabilization of compact Er:fiber laser technology at the full repetition rate of the oscillator. This approach readily enables us to generate widely tunable output in the entire visible and near infrared spectral regions [15,21], as well as synthesis of single-cycle light pulses [22] with absolute stability of the optical phase. Because of the capability of generating tailor-cut spectra in a broad frequency range [14], it becomes straightforward to seed high-power femtosecond amplifiers based on Yb: or Tm:fibers [23,24]. These technologies will give access to extreme nonlinear optics like the generation of high harmonics. As the allpassive approach provides extremely reliable and rigid locking of $f_{\mathrm{CEO}}$ on both short and long time scales, this system may also become an attractive seed source for Ti:sapphire technology, which is well established in attosecond science. To this end, the fundamental output at $1.55 \mu \mathrm{m}$ may be efficiently compressed to pulse durations below $30 \mathrm{fs}$ via soliton effects in standard telecom fibers [14] and then frequency doubled to center wavelengths of approximately $780 \mathrm{~nm}$. Finally, our compact system should also benefit applications in precision metrology with femtosecond frequency combs.

Support from EADS Astrium GmbH and TOPTICA Photonics AG is gratefully acknowledged.

\section{References}

1. G. G. Paulus, F. Grasbon, H. Walther, P. Villoresi, M. Nisoli, S. Stagira, E. Priori, and S. De Silvestri, Nature 414, 182 (2001).

2. M. Schultze, M. Fieß, N. Karpowicz, J. Gagnon, M. Korbman, M. Hofstetter, S. Neppl, A. L. Cavalieri, Y. Komninos, Th. Mercouris, C. A. Nicolaides, R. Pazourek, S. Nagele, J. Feist, J. Burgdörfer, A. M. Azzeer, R. Ernstorfer, R. Kienberger, U. Kleineberg, E. Goulielmakis, F. Krausz, and V. S. Yakovlev, Science 328, 1658 (2010).

3. T. Brabec and F. Krausz, Rev. Mod. Phys. 72, 545 (2000).

4. T. Udem, R. Holzwarth, and T. W. Hänsch, Nature 416, 233 (2002).

5. S. Rausch, T. Binhammer, A. Harth, E. Schulz, M. Siegel, and U. Morgner, Opt. Express 17, 20282 (2009).

6. S. Koke, C. Grebing, H. Frei, A. Anderson, A. Assion, and G. Steinmeyer, Nat. Photon. 4, 462 (2010).

7. A. Baltuška, T. Fuji, and T. Kobayashi, Phys. Rev. Lett. 88, 133901 (2002).

8. C. Manzoni, G. Cerullo, and S. De Silvestri, Opt. Lett. 29, 2668 (2004).

9. C. Manzoni, C. Vozzi, E. Benedetti, G. Sansone, S. Stagira, O. Svelto, S. De Silvestri, M. Nisoli, and G. Cerullo, Opt. Lett. 31, 963 (2006).

10. T. Fuji, A. Apolonski, and F. Krausz, Opt. Lett. 29, 632 (2004).

11. T. Fuji, N. Ishii, C. Teisset, X. Gu, T. Metzger, A. Baltuska, N. Forget, D. Kaplan, A. Galvanauskas, and F. Krausz, Opt. Lett. 31, 1103 (2006).

12. J. Moses, S. Huang, K. Hong, O. Mücke, E. Falcão-Filho, A. Benedick, F. Ilday, A. Dergachev, J. Bolger, B. Eggleton, and F. Kärtner, Opt. Lett. 34, 1639 (2009).

13. F. Tauser, A. Leitenstorfer, and W. Zinth, Opt. Express 11, 594 (2003).

14. A. Sell, G. Krauss, R. Scheu, R. Huber, and A. Leitenstorfer, Opt. Express 17, 1070 (2009).

15. F. Tauser, F. Adler, and A. Leitenstorfer, Opt. Lett. 29, 516 (2004).

16. M. Bellini and T. W. Hänsch, Opt. Lett. 25, 1049 (2000).

17. R. Huber, A. Brodschelm, F. Tauser, and A. Leitenstorfer, Appl. Phys. Lett. 76, 3191 (2000).

18. T. Fuji, J. Rauschenberger, A. Apolonski, V. S. Yakovlev, G. Tempea, T. Udem, C. Gohle, T. W. Hänsch, W. Lehnert, M. Scherer, and F. Krausz, Opt. Lett. 30, 332 (2005).

19. A. Sell, R. Scheu, A. Leitenstorfer, and R. Huber, Appl. Phys. Lett. 93, 251107 (2008).

20. M. Kakehata, H. Takada, Y. Kobayashi, K. Torizuka, Y. Fujihara, T. Homma, and H. Takahashi, Opt. Lett. 26, 1436 (2001).

21. K. Moutzouris, F. Adler, F. Sotier, D. Träutlein, and A. Leitenstorfer, Opt. Lett. 31, 1148 (2006).

22. G. Krauss, S. Lohss, T. Hanke, A. Sell, S. Eggert, R. Huber, and A. Leitenstorfer, Nat. Photon. 4, 33 (2010).

23. F. Röser, J. Rothhard, B. Ortac, A. Liem, O. Schmidt, T. Schreiber, J. Limpert, and A. Tünnermann, Opt. Lett. 30, 2754 (2005).

24. F. Haxsen, D. Wandt, U. Morgner, J. Neumann, and D. Kracht, Opt. Lett. 35, 2991 (2010). 